\title{
Micromotion of Dental Implants: Basic Mechanical Considerations
}

\author{
Werner Winter, ${ }^{1}$ Daniel Klein, ${ }^{1}$ and Matthias Karl ${ }^{2}$ \\ ${ }^{1}$ Department of Mechanical Engineering, University of Erlangen-Nuremberg, Egerlandstraße 5, 91058 Erlangen, Germany \\ ${ }^{2}$ Department of Prosthodontics, University of Erlangen-Nuremberg, Glueckstraße 11, 91054 Erlangen, Germany
}

Correspondence should be addressed to Matthias Karl; matthias.karl@uk-erlangen.de

Received 13 August 2012; Accepted 23 September 2012

Academic Editor: Raju Adhikari

Copyright (C) 2013 Werner Winter et al. This is an open access article distributed under the Creative Commons Attribution License, which permits unrestricted use, distribution, and reproduction in any medium, provided the original work is properly cited.

Micromotion of dental implants may interfere with the process of osseointegration. Using three different types of virtual biomechanical models, varying contact types between implant and bone were simulated, and implant deformation, bone deformation, and stress at the implant-bone interface were recorded under an axial load of $200 \mathrm{~N}$, which reflects a common biting force. Without friction between implant and bone, a symmetric loading situation of the bone with maximum loading and displacement at the apex of the implant was recorded. The addition of threads led to a decrease in loading and displacement at the apical part, but loading and displacement were also observed at the vertical walls of the implants. Introducing friction between implant and bone decreased global displacement. In a force fit situation, load transfer predominantly occurred in the cervical area of the implant. For freshly inserted implants, micromotion was constant along the vertical walls of the implant, whereas, for osseointegrated implants, the distribution of micromotion depended on the location. In the cervical aspect some minor micromotion in the range of $0.75 \mu \mathrm{m}$ could be found, while at the most apical part almost no relative displacement between implant and bone occurred.

\section{Introduction}

Micromotion of dental implants has been defined as minimal displacement of an implant body relative to the surrounding tissue which cannot be recognized with the naked eye [1] (Figure 1). Various authors have shown that excessive micromotion may interfere with the process of osseointegration of dental implants [2, 3]. Although exact data are missing, it has been postulated that micromotion between implant and bone must not surpass a threshold value of 150 micrometer $(\mu \mathrm{m})$ for successful implant healing [4-6].

In traditional loading protocols, where implants are allowed to heal undisturbed for periods of several months, the issue of implant micromotion is of limited importance. With the advent of modern treatment concepts including early and immediate loading of dental implants [7, 8], with implants being restored early in the healing phase, the issue of implant micromotion has gained significant importance $[4,5]$.

Numerous reports trying to relate clinical parameters to the phenomenon of implant micromotion can be found in the dental literature [8-11]. The nonuniform nomenclature, the varying experimental settings, and the partially contradicting results presented on the one hand indicate the complexity of the topic but on the other hand emphasize the need for clarifying basic engineering principles.

From a biomechanical perspective, successful osseointegration of dental implants depends on the way mechanical stresses and strains are transferred to the surrounding bone and tissues. The multiple factors hereby affecting stress and strain transfer include the type of loading that occurs, the type of implant-bone interface being present, the length and diameter of the implant, implant geometry and its surface texture, and the quality and quantity of the surrounding bone [13-19]. Only by understanding the most critical of these variables, strategies for optimizing implant stabilization can be developed. For determining how implant mobility, often referred to as micromotion, relative motion, micromovement, and so forth, or implant loading affects bone response, a closer look at implant deformation, bone deformation, and stress or strain at the implant-bone interface is required [20]. 


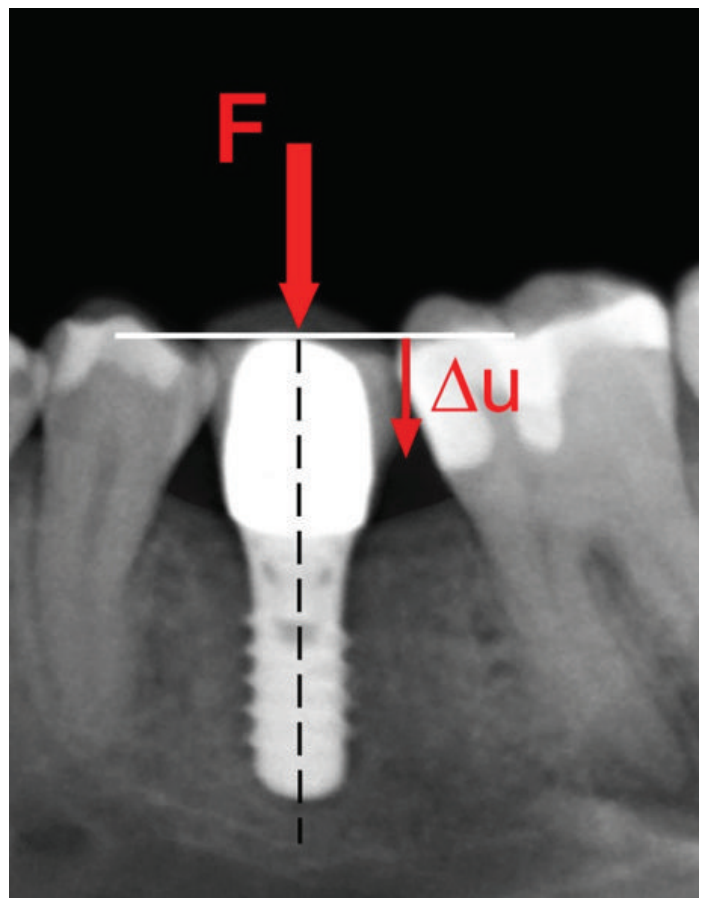

FIGURE 1: Single tooth implant used for replacing the first molar in the lower left mandible. An axial force acting on the occlusal surface of the restorations may displace the implant relative to the surrounding bone.

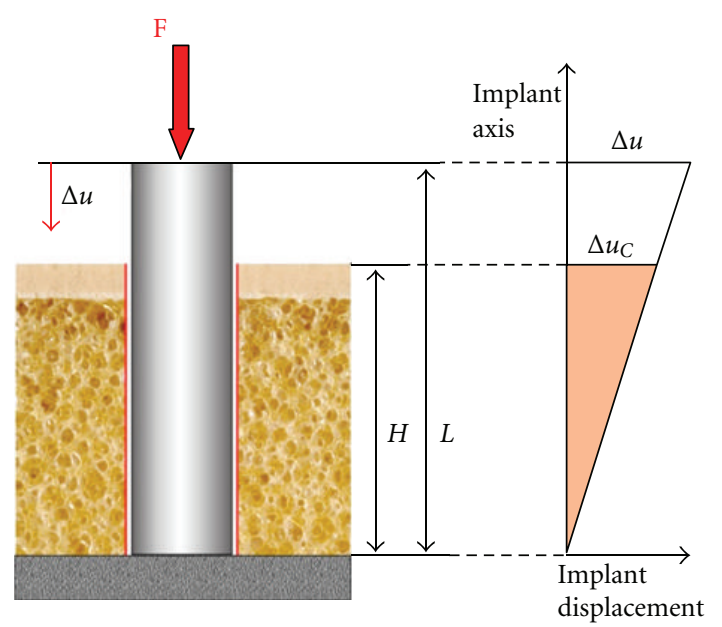

FIGURE 2: Description of scenario 1 with a dental implant resting on a fixed apical surface, with no contact existing between the vertical implant walls and the walls of the bony socket (left). When the implant is loaded vertically, deformation of the implant occurs mainly in the coronal part and decreases towards the apex. Similarly, relative displacement between implant and bone diminishes towards the apex (right).

In this context, it was the purpose of this paper to mechanically describe the phenomenon of micromotion occurring between implant and alveolar bone using simple spring models, continuum mechanics models, and 3DFinite-Element models simulating varying contact types between implant and bone [21-23].

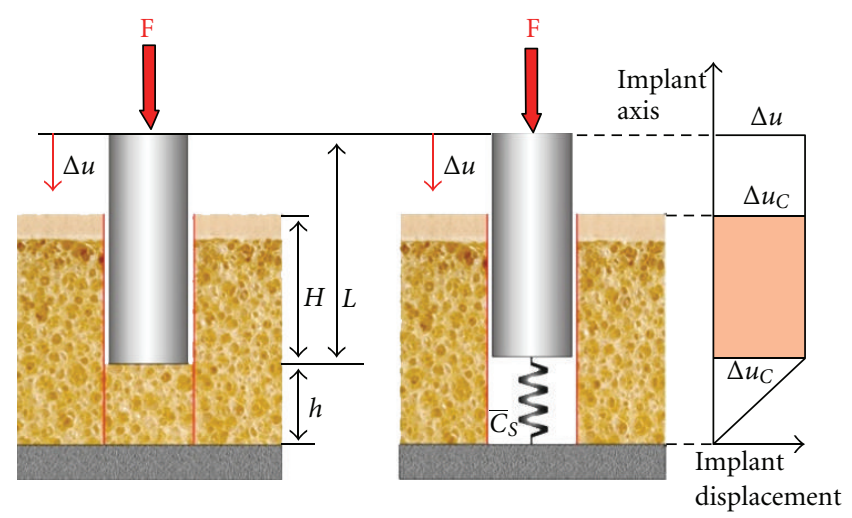

FIGURE 3: Description of scenario 2, where the implant rests on a layer of elastic trabecular bone with no contact existing between the vertical implant walls and the walls of the bony socket (left). The apically located layer of bone may be substituted by a spring which is compressed when an axial load is applied on the implant (center). Due to the great difference in elastic modulus between implant and trabecular bone, relative implant displacement is independent from the region of the implant considered (right).

\section{Material and Methods}

2.1. Basic Considerations. Three basic scenarios reflecting different anchoring situations of dental implants were considered. In scenario 1, the implant rests on an apically located fixed surface but neither has contact to cortical bone nor to trabecular bone at the vertical walls of the implant (Figure 2). In this situation, maximum implant deformation under 


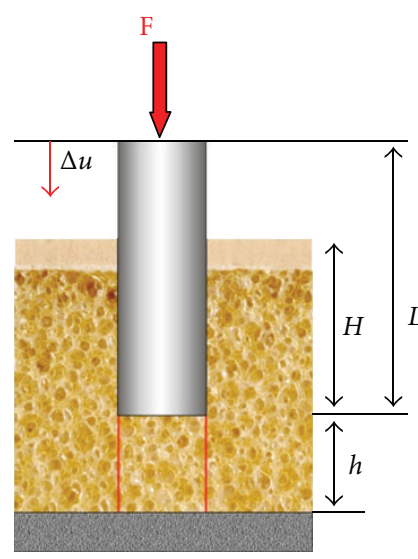

(a)

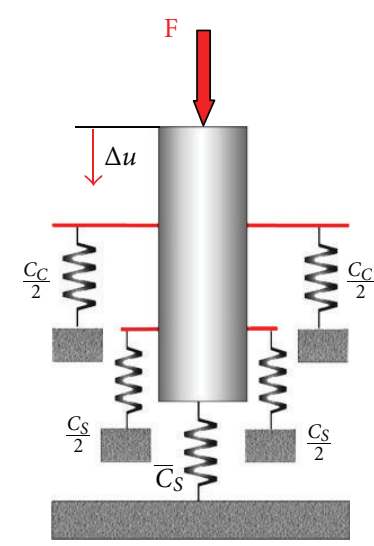

(b)

FIGURE 4: Scenario 3 showing an implant elastically supported by cortical and trabecular bone (a). The elastic support in the different regions can be replaced by a system of springs (b).

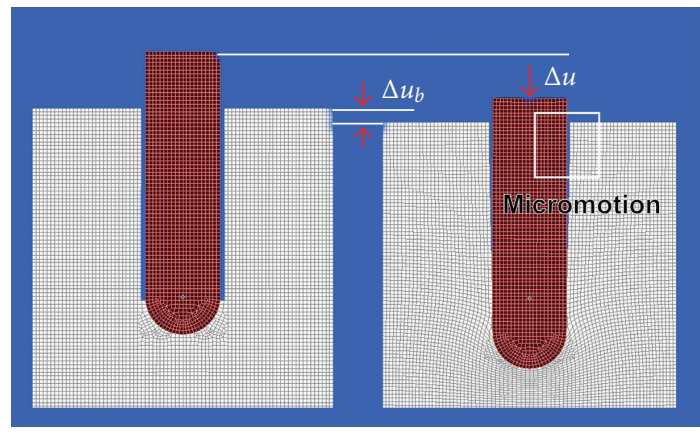

FIgURE 5: Without contact between implant and bone, an axial force acting on the implant causes implant dislocation as a result of elastic deformation of bone predominantly in the periapical region of the implant. Left: unloaded implant; right: loaded implant with implant displacement $\Delta u$ and displacement of cortical bone $\Delta u_{b}$ (displacement of a reference mark on bone).

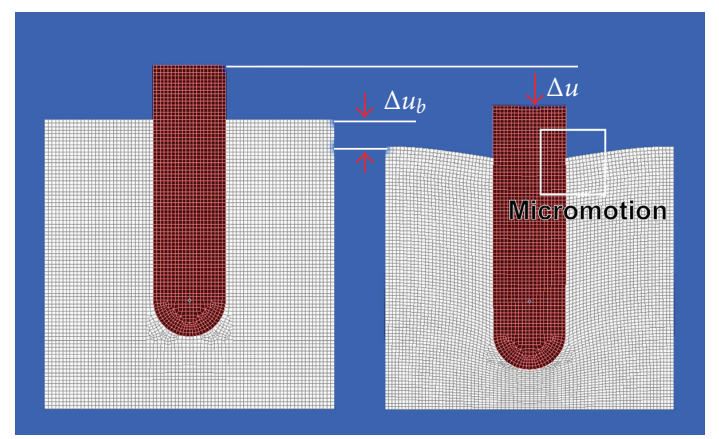

FIGURE 6: Considering an osseointegrated implant with contact between the implant surfaces and bone, axial implant loading causes elastic deformation of bone in all areas but no relative displacement between implant and bony socket, that is, no micromotion, occurs. Left: unloaded implant; right: loaded implant with implant displacement $\Delta u$ and displacement of cortical bone $\Delta u_{b}$ (displacement of a reference mark on bone). vertical loading occurs in the coronal part and diminishes gradually towards the apex. As a result, micromotion between the implant and the vertical walls of the socket also decreases towards the apical part of the implant. Axial deformation of the implant as a consequence of vertical loading can be calculated according to

$$
\Delta u=\frac{F}{E A / L}=\frac{F}{c}
$$

with $F$ standing for the vertical force applied, $E$ being the Young's modulus of the implant, $A$ being the cross section of the implant, $L$ being the length of the implant, and $c$ being the stiffness of the implant. Maximum micromotion $\Delta u_{c}$ at the cortical area can then be calculated according to

$$
\Delta u_{c}=\frac{\Delta u}{L} H
$$

with $H$ reflecting the height of cortical and trabecular bone around the implant.

For scenario 2, the fixed apical rest of the implant was altered by adding a layer of elastic trabecular bone apically to the implant. Here, an axial force acting on the implant predominantly causes compression of the elastic material the implant is resting on. Due to the drastically smaller elastic modulus of trabecular bone as compared to titanium, the deformation of the implant can be neglected and the relative movement between implant and bone is independent from the region of the implant considered (Figure 3). In this situation, implant displacement may be calculated according to

$$
\Delta u=\frac{F}{E_{s} A / h}\left[1+\frac{L}{h} \frac{E_{s}}{E}\right]=\frac{F}{\overline{C_{s}}}[1+\beta],
$$

where $E_{s}$ is the Young's modulus of the trabecular bone and $h$ the height of the bone underneath the implant.

Taking into account that the Young's modulus of the implant is much greater than the Young's modulus of the 

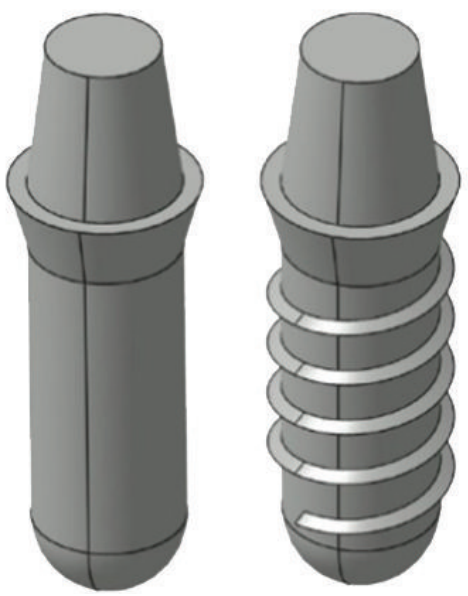

(a)
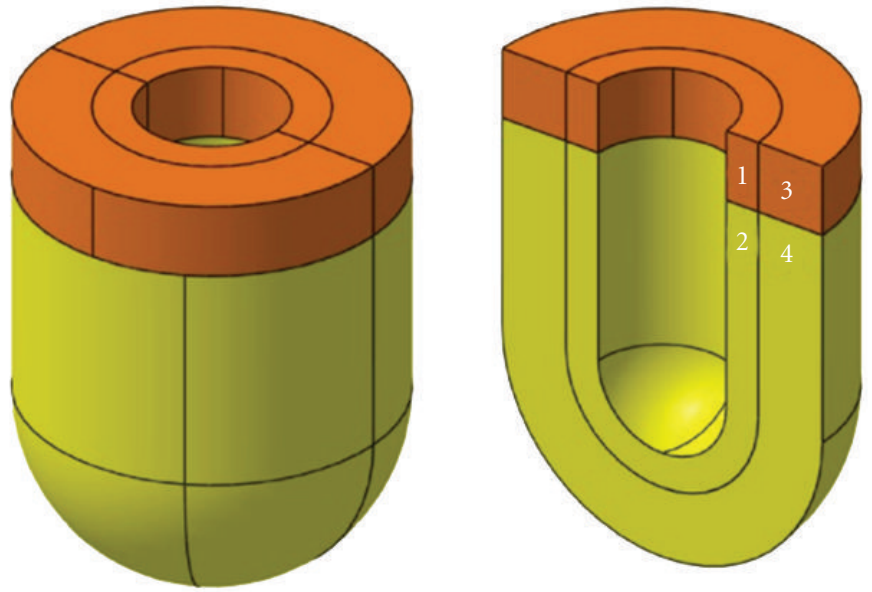

(b)

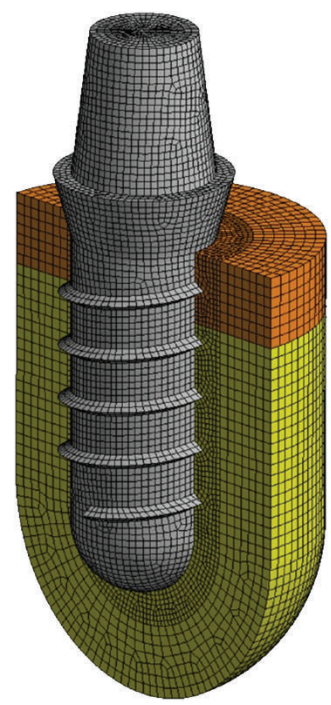

(c)

Figure 7: (a) Three-dimensional finite element models of dental implants with and without threads [12]. (b) Three-dimensional finite element model of a bony implant socket with cortical and trabecular bone. Areas (1) and (2) surrounding the implant are designed as an intermediate layer allowing the elastic modulus to be set independently from areas (3) and (4) representing native bone which is not affected by healing processes occurring during osseointegration [12]. (c) Three-dimensional finite element model of a single implant embedded in a bone segment consisting of cortical and trabecular bone (calculations were done on a complete model; for illustration purposes the model is cut in half) [12].

trabecular bone $\left(E \gg E_{s}\right)$ it is accepted $\beta \ll 1$ and furthermore the approximation for the micromotion

$$
\Delta u_{c}=\Delta u \approx \frac{F}{\overline{C_{s}}} .
$$

Consequently, micromotion at the cortical area of the implant and the relative micromotion between implant and bone are identical, both being related to the stiffness $\overline{C_{s}}$ of the trabecular bone underneath the implant.

Further approximating the clinical situation of an osseointegrated implant, in scenario 3 the implant is elastically supported by surrounding cortical and trabecular bone. Due to the fixed contact between implant and bone, micromotion at this interface does not occur, when the implant is axially loaded (Figure 4). Neglecting the deformation of the implant $\left(E \gg E_{s}\right)$ the implant displacement may be calculated according to

$$
\Delta u=\frac{F}{\overline{C_{s}}} \frac{1}{\left(1+\left(C_{s} / \overline{C_{s}}\right)+\left(C_{c} / \overline{C_{s}}\right)\right)} .
$$

Under the circumstances of scenario 3, no relative micromotion between implant and bone exists. The displacement of the implant equals the micromotion depending on the stiffness of both cortical and trabecular bone surrounding the implant.

Further approximating clinical reality, continuum mechanics models were considered revealing implant displacement due to elastic deformation of bone when no 


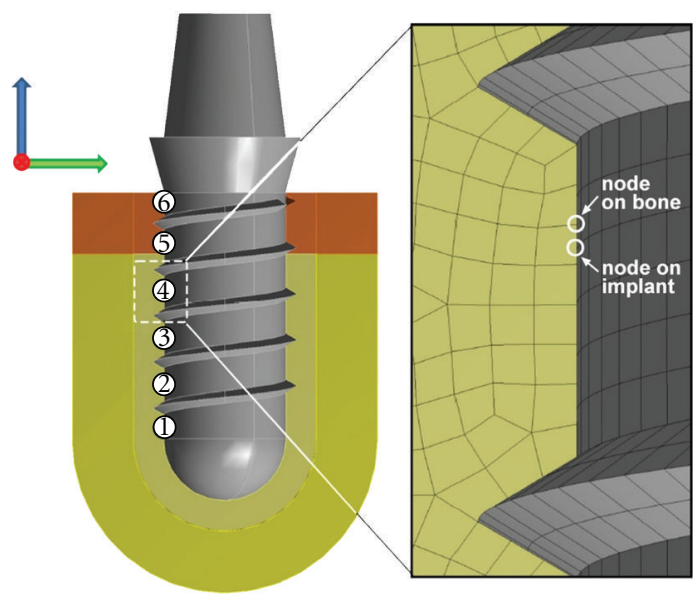

FIgURE 8: Definition of micromotion at the implant bone interface. Six corresponding nodes on the implant and on the bone were used as reference marks. For determining the relative displacement of two corresponding nodes on bone and implant, the displacement of a specific reference mark on the bone was subtracted from the displacement of the corresponding reference mark on the implant.

contact between implant and bone was modelled in a plane strain FE-model (Figure 5). This model correlates with scenario 2 described above (Figure 3). Simulating contact between implant and bone, bone is also elastically deformed in the cervical portion of the implant when an axial load is exerted; however, no relative micromotion between implant and bone occurs at the interface (Figure 6), and the implant displacement (micromotion) is related to the elastic properties of the cortical and trabecular bone.

2.2. Finite Element Analysis. For a more realistic representation of clinical conditions, three-dimensional FE models [12] of dental implants with and without threads were generated (Figure 7(a)) which were subsequently embedded in a bony socket consisting of cortical and trabecular bone and an intermediate layer surrounding the implant (Figure 7(b)). The geometry of the models was generated with a CAD Program (SolidWorks 2011, SolidWorks Deutschland $\mathrm{GmbH}$, Haar, Germany) and imported in a FE program (ANSYS Workbench 12, ANSYS Inc., Canonsburg, PA, USA).

Combining both components, three-dimensional $\mathrm{FE}$ models (Figure 7(c)) were obtained for evaluating micromotion between implant and bone when an axial vertical force of $200 \mathrm{~N}$ was exerted which reflects an average biting force $[24,25]$. Different stages of osseointegration were simulated by altering the elastic modulus of the intermediate bone layer [21-23]. The contact type between implant and bone could be modified as friction free, only transferring compressive forces and allowing for sliding and gap formation, to friction (friction coefficient 0.3 ) and force fit, respectively [21].

In general, isotropic linear model parameters were applied, defining the contact type between the different layers of bone as "bond." Out of the large number of possible solutions for solving contact problems, the augmented Lagrange method was chosen as accompanying optimization
TABLE 1: Material properties (Young's moduli in MPa) chosen in the different models. Poisson's ratio is 0.3 for all materials.

\begin{tabular}{lcc}
\hline Structure & $\begin{array}{c}\text { Osseointegrated } \\
\text { implant }\end{array}$ & Healing state \\
\hline Cortical bone & 14000 & 14000 \\
Trabecular bone & 3000 & 3000 \\
Implant & 110000 & 110000 \\
Intermediate layer-cortical area & 14000 & 1000 \\
Intermediate layer-trabecular area & 3000 & 1000 \\
\hline
\end{tabular}

method. This method was applied for defining all contacts not allowing contacting components to penetrate each other. Poisson's ratio was set at 0.3 for all materials. Based on the results of previous investigations $[22,23]$ indicating that the size of the models was sufficient for evaluating micromotion, model dimensions were reduced to a minimum and the borders of the models were fixed. Depending on model type, 160000 hexaeder elements and 600000 to 650000 nodes were used to set up the models using the elastic modules given in Table 1. Based on the fact that the elastic values and the strength limits of biologic materials in vivo-such as the bone-implant interface-are highly complex [24], only two states of osseointegration were considered (starting point and end point of osseointegration). These different states were modelled by different elastic values in the areas (1) and (2) in Figure 7(b).

Results of all simulations were recorded as von Mises equivalent stress in addition to contour plots of global displacement.

For calculating relative displacement between implant and bone (relative micromotion), a total of six corresponding nodes at the implant bone interface were established as reference marks. As the displacement of a specific reference mark on the implant represents both displacement of bone and implant, the displacement of the corresponding reference mark on the bone (Figure 8) was subtracted.

\section{Results}

Simulating $200 \mathrm{~N}$ axial force acting on an osseointegrated cylindrical implant with no friction between implant and bone caused a symmetric loading situation of the bone surrounding the implant with maximum loading and maximum displacement occurring at the apical part of the implant (Figures 9(a) and 10(a)).

Adding threads to the implant led to a decrease both in loading and displacement occurring at the apical part of the implant. Simultaneously, greater distribution of loading and displacement was observed at the vertical walls of the implants (Figures 9(b) and 10(b)).

Introducing friction between implant and bone (Figures 9(c) and 10(c)) further decreased global displacement and resulted in a more homogeneous distribution of loads as compared to the force fit situation (Figures 9(d) and 10(d)), where load transfer predominantly occurred in the cervical area of the implant, where cortical bone was modelled. 


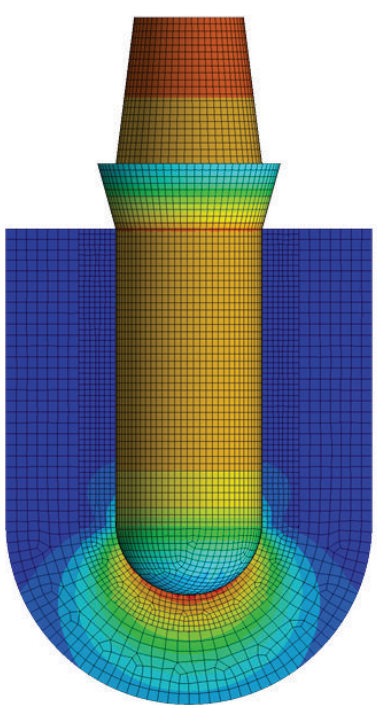

(a)

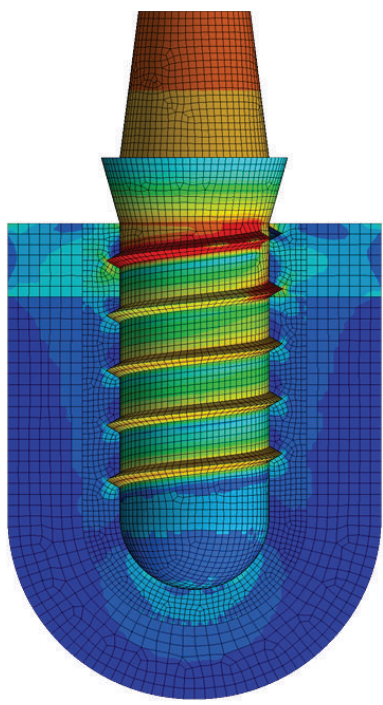

(c)

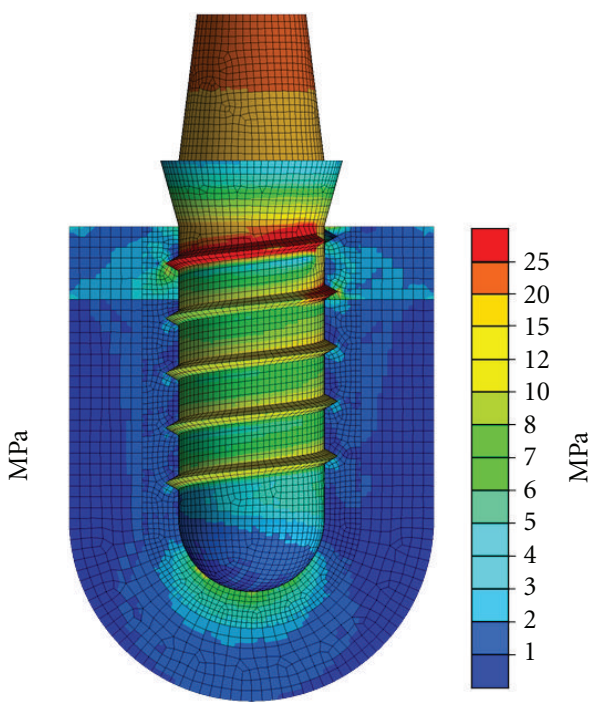

(b)

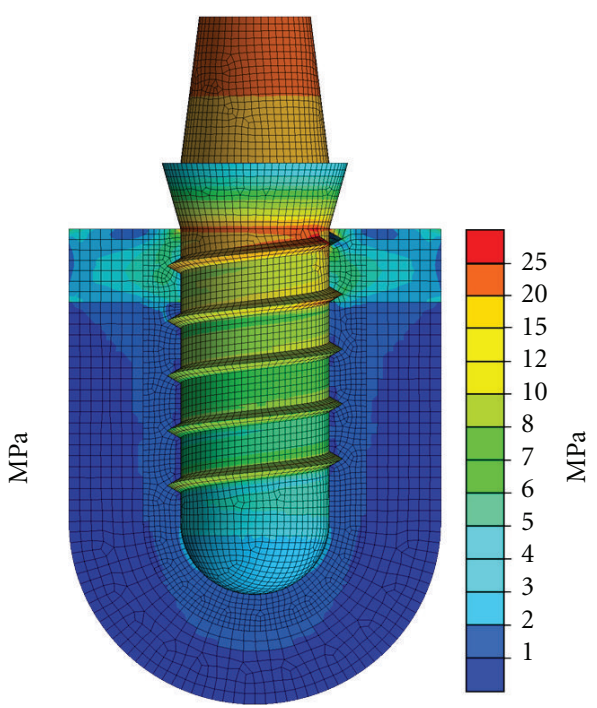

(d)

Figure 9: Distribution of von Mises equivalent stress around implants loaded with $200 \mathrm{~N}$ axial vertical force [12]: cylindrical implant without friction between implant and bone (a), threaded implant without friction between implant and bone (b), threaded implant with friction between implant and bone (coefficient of friction: 0.3) (c), and threaded implant with force fit between implant and bone (d).

For freshly inserted implants with a soft intermediate layer of bone modelled around the implants, the introduction of a friction coefficient led to a considerable reduction in micromotion between implant and bone as well as to reduced displacement of all reference marks on the implant. Displacement of the reference marks on the bone remained on a constant level. Overall, comparable values for micromotion were recorded at all corresponding reference marks (Figure 11).

Simulating an osseointegrated implant in general reduced all displacement values by about $50 \%$ compared to the situation of a freshly inserted implant. Again the introduction of a friction coefficient led to a considerable reduction in micromotion between implant and bone as well as to reduced displacement of all reference marks on the implant.
Displacement of the reference marks on the bone remained on a constant level. In contrast to a freshly inserted implant, the distribution of micromotion depended on the location of the reference mark. Whereas in the cervical aspect some minor micromotion in the range of $0.75 \mu \mathrm{m}$ could be found, at the most apical reference almost no relative displacement between implant and bone occurred (Figure 12).

\section{Discussion}

Within the limitations of this investigation, the effect of friction phenomena and implant design (cylindrical versus threaded) on stress distribution and implant displacement could be demonstrated. Both the introduction of friction between implant and bone as well as the addition of threads 


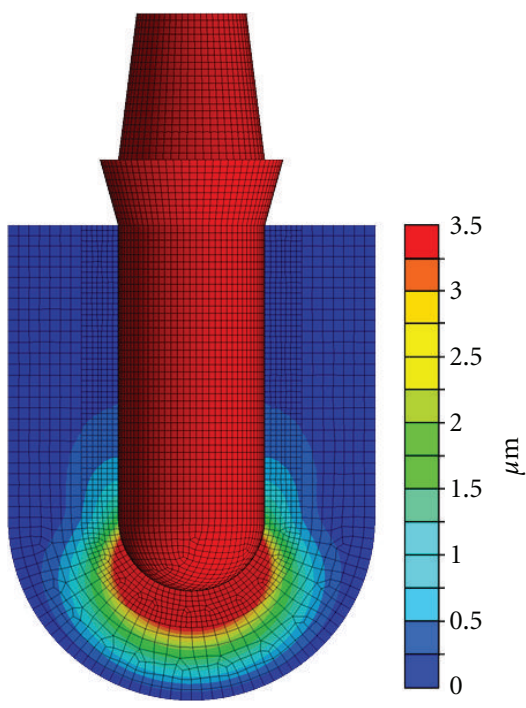

(a)

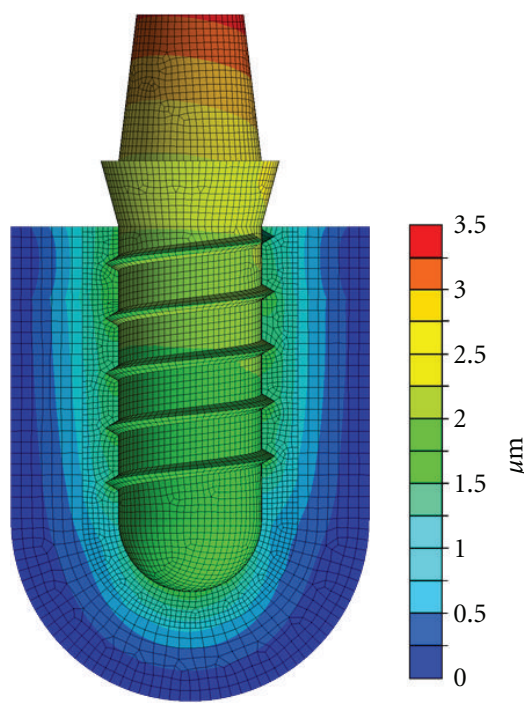

(c)

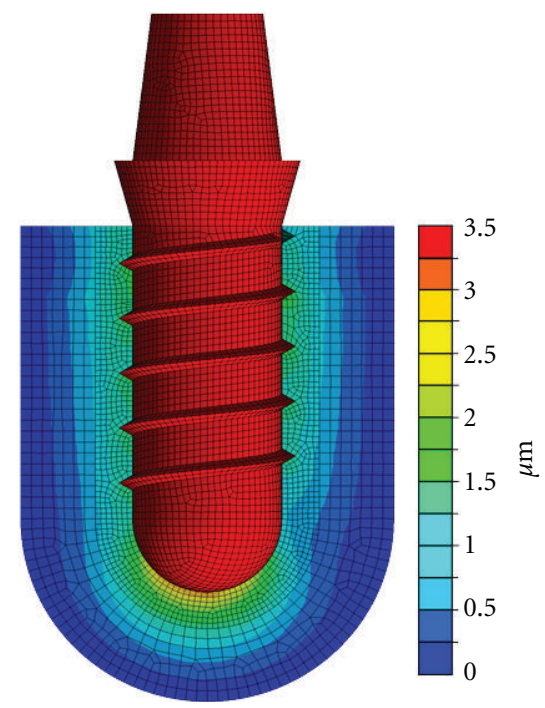

(b)

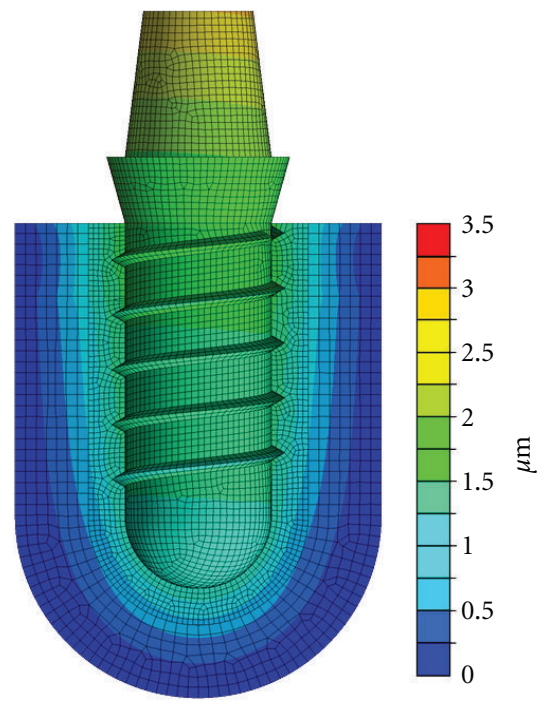

(d)

Figure 10: Distribution of global displacement around implants loaded with $200 \mathrm{~N}$ axial vertical force [12]: cylindrical implant without friction between implant and bone (a), threaded implant without friction between implant and bone (b), threaded implant with friction between implant and bone (coefficient of friction: 0.3) (c), and threaded implant with force fit between implant and bone (d).

to a cylindrically shaped implant resulted in the reduction of implant displacement under an axial load of $200 \mathrm{~N}$. Simultaneously, a more homogeneously distributed loading situation at the implant bone interface could be observed. Changing the contact type between implant and bone to force fit resulted in load transfer predominantly occurring in the cervical part of the implant surrounded by stiffer cortical bone. This is in strict contrast to a situation with no friction modelled resulting in maximum loading of bone surrounding the periapical region of the implant. From a clinical perspective, these findings indicate that screw-shaped implants are advantageous while bone quality probably plays the most important role in achieving sufficient primary implant stability for immediate loading. All these factors should be taken into account when choosing a specific loading protocol.

Based on a comparison of freshly inserted and osseointegrated implants it could be shown that the healing status affects the occurrence of micromotion phenomena along the implant bone interface. For a soft implant bone interface, reflecting early stages of osseointegration, micromotion remained on a constant level regardless of the location considered. Simulating mature bone reflecting an osseointegrated implant, the introduction of a friction coefficient between implant and bone dramatically changed the distribution of micromotion along the implant bone interface. In addition to generally reduced levels of micromotion as 


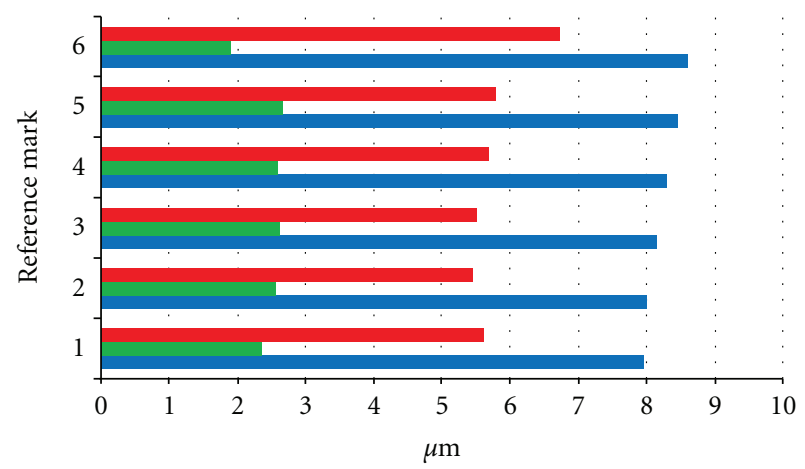

Micromotion

Displacement of reference mark on bone

Displacement of reference mark on implant

(a)

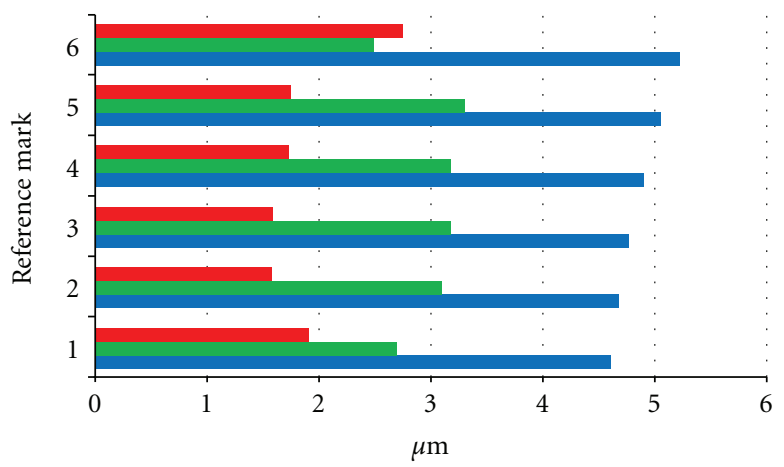

Micromotion

Displacement of reference mark on bone

Displacement of reference mark on implant

(b)

FIGURE 11: Displacement of corresponding reference marks on bone and implant for freshly inserted implants and resulting micromotion: data recorded from model without friction between bone and implant (a), data recorded from model with friction between bone and implant (b) (note the different scales).

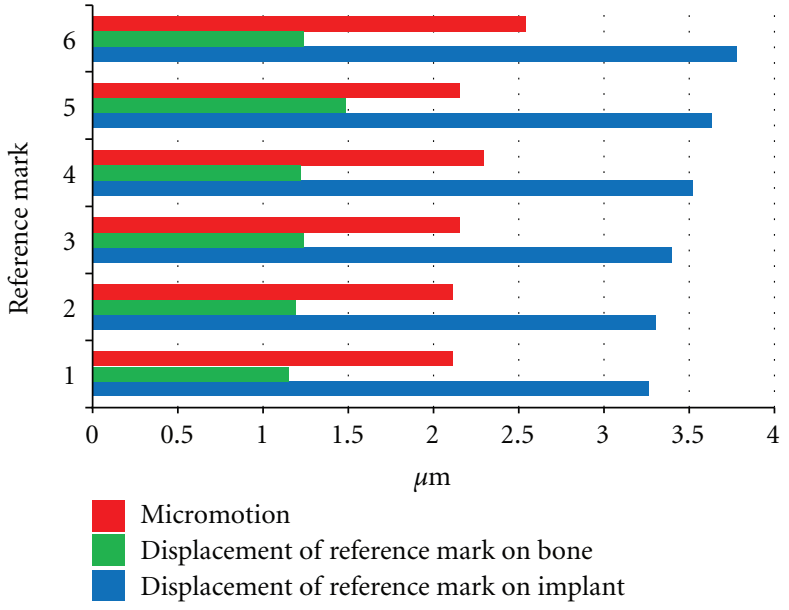

(a)

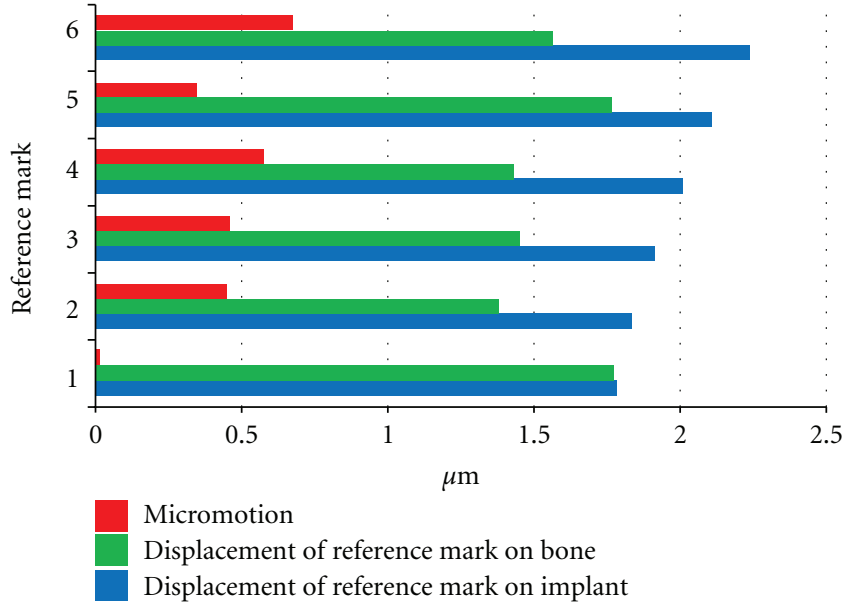

(b)

FIGURE 12: Displacement of corresponding reference marks on bone and implant for osseointegrated implants and resulting micromotion: data recorded from model without friction between bone and implant (a), data recorded from model with friction between bone and implant (b), note the different scales!

compared to a freshly inserted implant, a decrease in micromotion was noted. The amount of micromotion decreased towards the apex of the implant.

It may be seen as a limitation of this study that only one specific value for axial loading of the implants was chosen. Based on studies by Brunski and coworkers [20], axial components of biting forces can range from 100 to $2400 \mathrm{~N}$, while the exact values depend on factors such as location in the mouth and nature of food. For patients having implantsupported dentures, axial closure forces ranging from 45 to $255 \mathrm{~N}$ have been reported [25]. It thus appears that the value chosen reflects clinical loading magnitudes.

Furthermore, besides the pure mechanical aspects addressed in this paper, also biologic factors play an important role in the process of osseointegration of dental implants. Following implant placement, the healing period starts with the adherence of serum proteins, followed by the attachment and proliferation of mesenchymal cells. Consequently, osteoid is formed in what is then mineralized. From then onwards, bone remodeling occurs as an adaptation to the implants environment [26]. With these processes occurring simultaneously to mechanical loading in an immediate loading situation, the interaction of both mechanical and biologic factors seems to be critical to the integration of the implant.

\section{Conclusions}

Given the nonuniform distribution of micromotion between implant and bone, it appears questionable whether currently 
available methods for experimentally determining this phenomenon provide meaningful data. The only valid approach for evaluating micromotion phenomena at the implant-bone interface appears to be finite element analysis. However, care has to be taken to set proper materials and interface characteristics as these parameters may greatly influence the outcome.

\section{Acknowledgment}

Figures 7(a), 7(b), 7(c); 9(a)-9(d): 10(a)-10(d) have been previously published in [12] and are reused with permission from Allen Press Publishing Services.

\section{References}

[1] W. R. Laney, Glossary of Oral and Maxillofacial Implants, German edition, Quintessenz, Berlin, Germany, 2008.

[2] J. B. Brunski, "The influence of force, motion and related quantities on the response of bone to implant," in Non-Cemented Total Hip Arthroplasty, R. Fitzgerald, Ed., pp. 7-21, Ravens Press, New York, NY, USA, 1988.

[3] J. B. Brunski, "In vivo bone response to biomechanical loading at the bone/dental-implant interface," Advances in Dental Research, vol. 13, pp. 99-119, 1999.

[4] S. Szmukler-Moncler, H. Salama, Y. Reingewirtz, and J. H. Dubruille, "Timing of loading and effect of micromotion on bone-dental implant interface: review of experimental literature," Journal of Biomedical Materials Research, vol. 43, pp. 192-203, 1998.

[5] S. Szmukler-Moncler, A. Piattelli, G. A. Favero, and J. H. Dubruille, "Considerations preliminary to the application of early and immediate loading protocols in dental implantology," Clinical Oral Implants Research, vol. 11, no. 1, pp. 12-25, 2000.

[6] H. Kawahara, D. Kawahara, M. Hayakawa, Y. Tamai, T. Kuremoto, and S. Matsuda, "Osseointegration under immediate loading: biomechanical stress-strain and bone formation-resorption," Implant Dentistry, vol. 12, no. 1, pp. 61-68, 2003.

[7] G. E. Romanos, "Present status of immediate loading of oral implants," The Journal of Oral Implantology, vol. 30, no. 3, pp. 189-197, 2004.

[8] P. Trisi, G. Perfetti, E. Baldoni, D. Berardi, M. Colagiovanni, and G. Scogna, "Implant micromotion is related to peak insertion torque and bone density," Clinical Oral Implants Research, vol. 20, no. 5, pp. 467-471, 2009.

[9] W. Engelke, O. A. Decco, M. J. Rau, M. C. A. Massoni, and W. Schwarzwäller, "In vitro evaluation of horizontal implant micromovement in bone specimen with contact endoscopy," Implant Dentistry, vol. 13, no. 1, pp. 88-94, 2004.

[10] S. Holst, H. Geiselhoeringer, M. Wichmann, and A. I. Holst, "The effect of provisional restoration type on micromovement of implants," Journal of Prosthetic Dentistry, vol. 100, no. 3, pp. 173-182, 2008.

[11] M. Goellner, J. Schmitt, M. Karl, M. Wichmann, and S. Holst, "The effect of axial and oblique loading on the micromovement of dental implants," International Journal of Oral \& Maxillofacial Implants, vol. 26, pp. 257-264, 2011.

[12] W. Winter, D. Klein, and M. Karl, "Effect of model parameters on finite element analysis of micromotions in implant dentistry," The Journal of Oral Implantology. In press.
[13] J. E. Bechtold, O. Mouzin, L. Kidder, and K. Søballe, "A controlled experimental model of revision implants: Part II. Implementation with loaded titanium implants and bone graft," Acta Orthopaedica Scandinavica, vol. 72, no. 6, pp. 650-656, 2001.

[14] L. C. Jones, C. Frondoza, and D. S. Hungerford, "Effect of PMMA particles and movement on an implant interface in a canine model," Journal of Bone and Joint Surgery B, vol. 83, no. 3, pp. 448-458, 2001.

[15] O. Mouzin, K. Soballe, and J. E. Bechtold, "Loading improves anchorage of hydroxyapatite implants more than titanium implants," Journal of Biomedical Materials Research, vol. 58, pp. 61-68, 2001.

[16] R. Skripitz and P. Aspenberg, "Early effect of parathyroid hormone (1-34) on implant fixation," Clinical Orthopaedics and Related Research, no. 392, pp. 427-432, 2001.

[17] S. Toksvig-Larsen, L. P. Jorn, L. Ryd, and A. Lindstrand, "Hydroxyapatite-enhanced tibial prosthetic fixation," Clinical Orthopaedics and Related Research, no. 370, pp. 192-200, 2000.

[18] H. M. Van Der Vis, P. Aspenberg, R. K. Marti, W. Tigchelaar, and C. J. F. Van Noorden, "Fluid pressure causes bone resorption in a rabbit model of prosthetic loosening," Clinical Orthopaedics and Related Research, no. 350, pp. 201-208, 1998.

[19] H. G. Willert and G. H. Buchhorn, "Osseointegration of cemented and noncemented implants in artificial hip replacement: long-term findings in man," Journal of Long-Term Effects of Medical Implants, vol. 9, no. 1-2, pp. 113-130, 1999.

[20] J. Brunski, J. Currey, J. A. Helms, P. Leucht, A. Nanci, and R. Wazen, "Implant geometry, interfacial strain and mechanobiology of oral implants revisited," in Proceedings of the first PI Branemark Scientific Symposium, Gothenburg 2009, R. Gottlander and D. van Steenberghe, Eds., pp. 45-59, Quintessence Publishing, London, UK, 2011.

[21] D. C. Holmes and J. T. Loftus, "Influence of bone quality on stress distribution for endosseous implants," The Journal of Oral Implantology, vol. 23, no. 3, pp. 104-111, 1997.

[22] W. Winter, S. Möhrle, S. Holst, and M. Karl, "Parameters of implant stability measurements based on resonance frequency and damping capacity: a comparative finite element analysis," The International Journal of Oral \& Maxillofacial Implants, vol. 25 , no. 3, pp. 532-539, 2010.

[23] W. Winter, P. Steinmann, S. Holst, and M. Karl, "Effect of geometric parameters on finite element analysis of bone loading caused by nonpassively fitting implant-supported dental restorations," Quintessence International, vol. 42, pp. 471-478, 2011.

[24] J. B. Brunski, "Biomechanical factors affecting the bone-dental implant interface," Clinical Materials, vol. 10, no. 3, pp. 153-201, 1992.

[25] A. B. Carr and W. R. Laney, "Maximum occlusal force levels in patients with osseointegrated oral implant prostheses and patients with complete dentures," The International Journal of Oral \& Maxillofacial Implants, vol. 2, no. 2, pp. 101-108, 1987.

[26] S. Raghavendra, M. C. Wood, and T. D. Taylor, "Early wound healing around endosseous implants: a review of the literature," International Journal of Oral and Maxillofacial Implants, vol. 20, no. 3, pp. 425-431, 2005. 

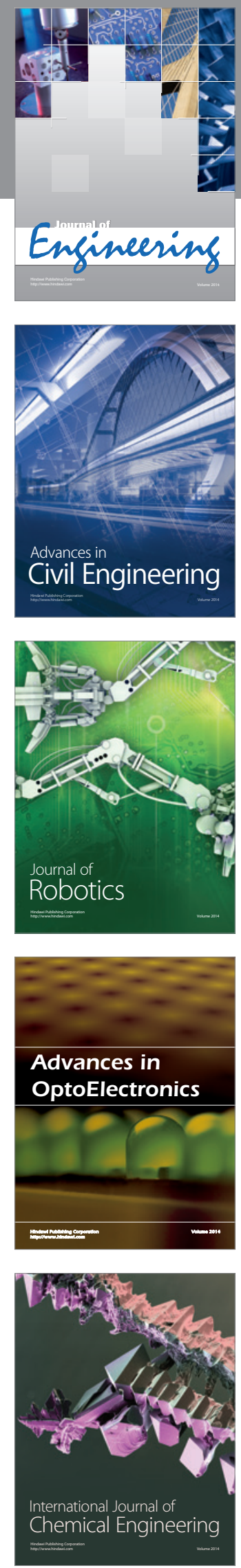

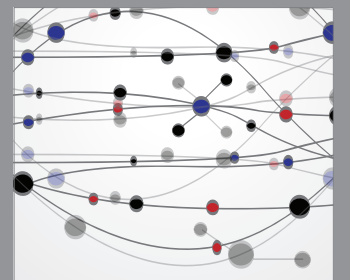

The Scientific World Journal
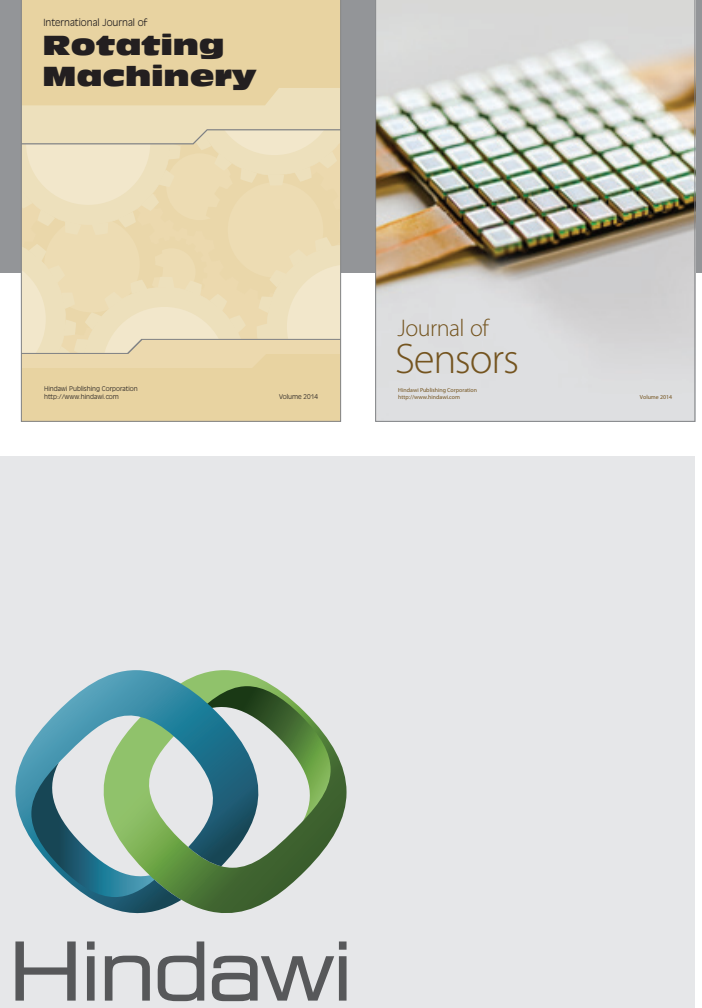

Submit your manuscripts at http://www.hindawi.com
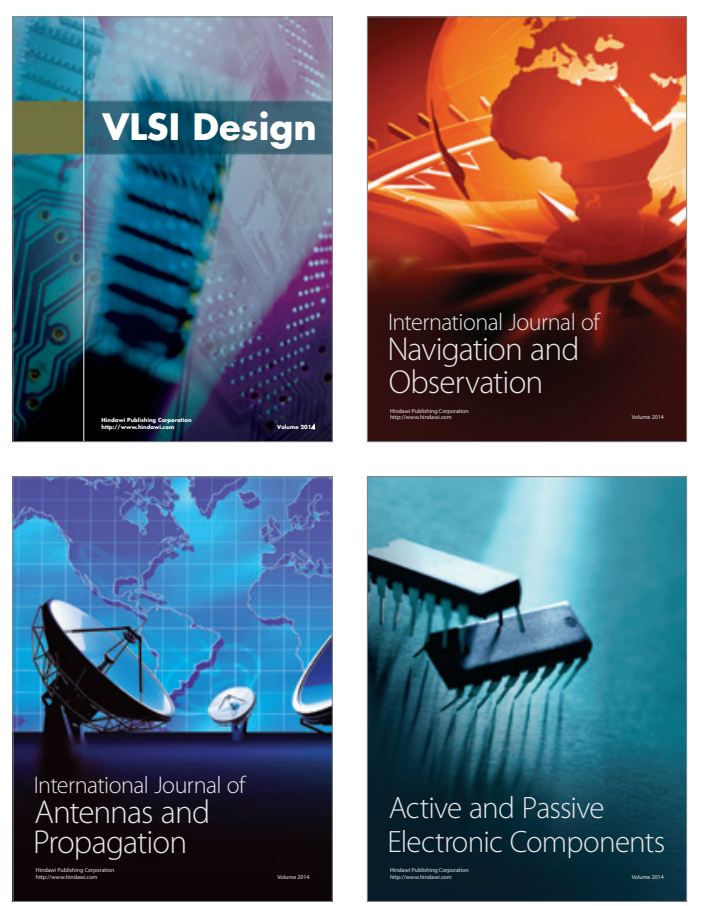
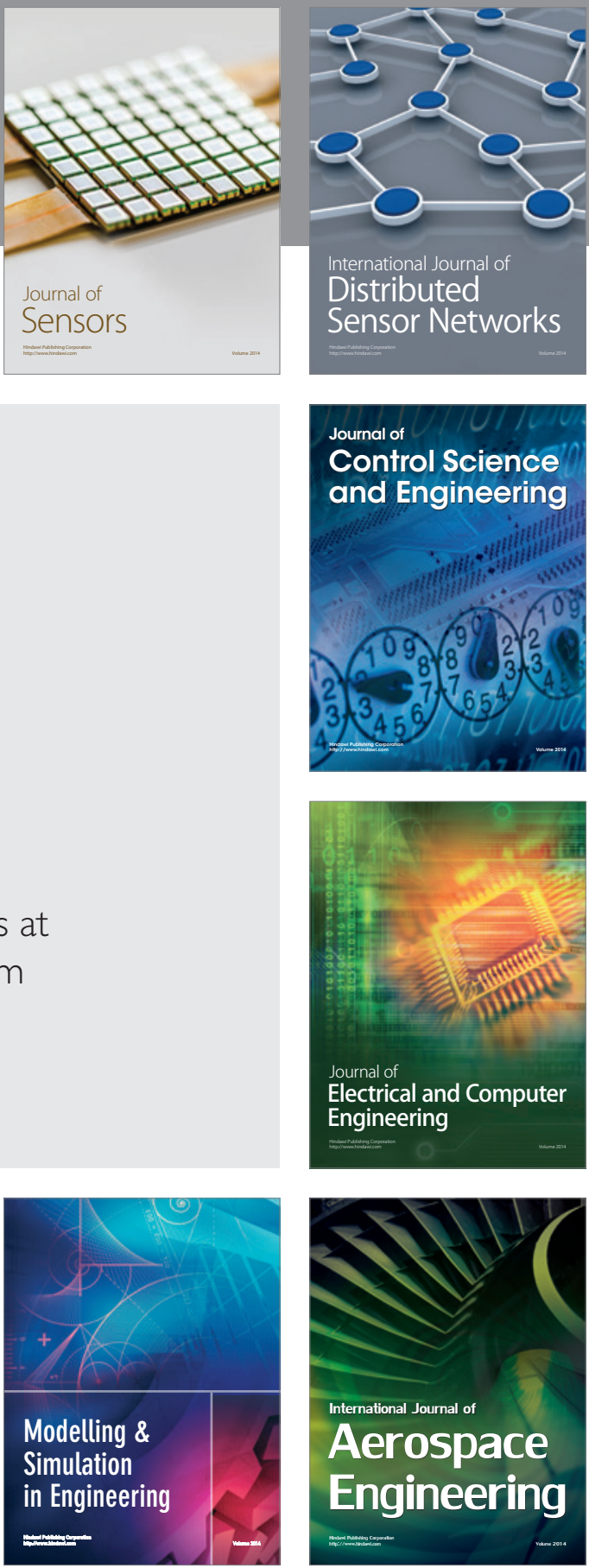

Journal of

Control Science

and Engineering
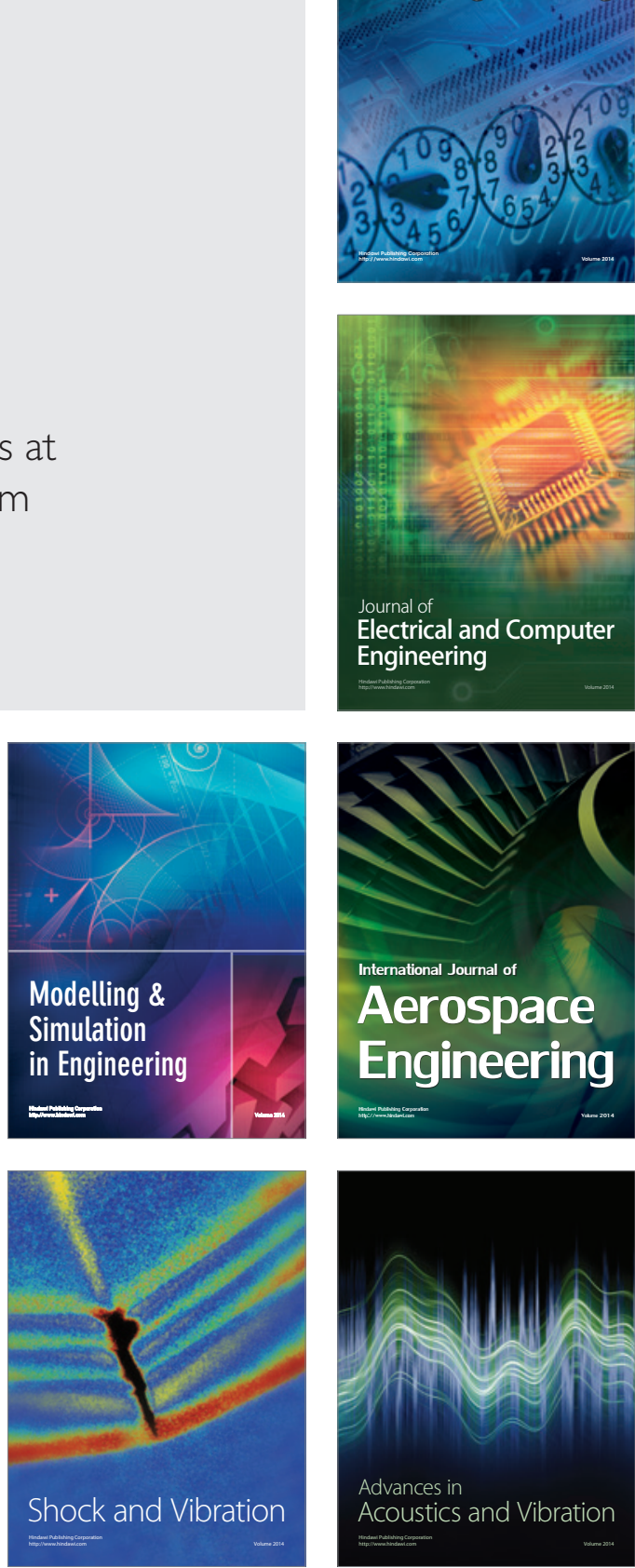